\title{
Integrable Solutions of a Nonlinear Integral Equation via Noncompactness Measure and Krasnoselskii's Fixed Point Theorem
}

\author{
Mahmoud Bousselsal ${ }^{1,2}$ and Sidi Hamidou $\mathrm{Jah}^{2}$ \\ ${ }^{1}$ Laboratoire d'Analyse Nonlineaire et HM, Department of Mathematics, ENS-Kouba, Vieux-Kouba 16250, Algiers, Algeria \\ ${ }^{2}$ Department of Mathematics, College of Science, Qassim University, P.O. Box 6644, Buraydah 51452, Saudi Arabia
}

Correspondence should be addressed to Mahmoud Bousselsal; bousselsal55@gmail.com

Received 20 September 2013; Revised 2 January 2014; Accepted 12 January 2014; Published 16 March 2014

Academic Editor: Seenith Sivasundaram

Copyright (C) 2014 M. Bousselsal and S. H. Jah. This is an open access article distributed under the Creative Commons Attribution License, which permits unrestricted use, distribution, and reproduction in any medium, provided the original work is properly cited.

We study the existence of solutions of a nonlinear Volterra integral equation in the space $L^{1}[0,+\infty)$. With the help of Krasnoselskii's fixed point theorem and the theory of measure of weak noncompactness, we prove an existence result for a functional integral equation which includes several classes on nonlinear integral equations. Our results extend and generalize some previous works. An example is given to support our results.

\section{Introduction}

In this paper, we present an existence result for the functional integral equation

$$
\begin{aligned}
x(t)= & g\left(t, x(t), x\left(\psi_{1}(t)\right)\right) \\
& +f\left(t, \int_{0}^{\psi_{2}(t)} u\left(t, s, x\left(\psi_{3}(s)\right)\right) d s,\right. \\
& \left.\int_{0}^{\infty} v\left(t, s, x\left(\psi_{4}(s)\right)\right) d s\right),
\end{aligned}
$$

$t \geq 0$, where $f, g, u$, and $v$ are given measurable functions while $x(t) \in L^{1}\left(\mathbb{R}_{+}\right)$is an unknown function.

Equation (1) is a general form of many integral equations, such as the mixed Volterra-Fredholm integral equation

$$
\begin{aligned}
x(t)= & g(t)+\int_{0}^{t} k(t, s) u(s, x(s)) d s \\
& +\alpha(t) \int_{0}^{\infty} v(s, x(s)) d s, \quad t \geq 0
\end{aligned}
$$

which has been considered by many authors, see for example, $[1-3]$ and references cited therein. Moreover, (1) contains the nonlinear Volterra and Fredholm integral equation on $\mathbb{R}_{+}$ such as

$$
x(t)=g(t)+\int_{0}^{t} u(t, s, x(s)) d s, \quad t \geq 0
$$

which is studied in $[4,5]$. The existence of solution of Urysohn's equation,

$$
x(t)=F\left(t, x(t), \int_{0}^{\infty} u(t, s, x(s)) d s\right), \quad t \geq 0
$$

was studied in [6] where he proved that (4) has a solution $x(t)$ in $C_{0}\left(\mathbb{R}_{+}\right)$space. The problem

$$
\begin{array}{r}
x(t)=f\left(t, \int_{0}^{t} u(t, s, x(s)) d s, \int_{0}^{\infty} \alpha(t) v(s, x(s)) d s\right), \\
t \geq 0,
\end{array}
$$


was studied in [1] where they obtained the existence of solution $x(t) \in L^{1}\left(\mathbb{R}_{+}\right)$by using the classical Schauder fixed point principle. The nonlinear integral equation

$$
\begin{aligned}
x(t)= & g\left(t, x\left(\psi_{1}(t)\right)\right) \\
& +f\left(t, \int_{0}^{\psi_{2}(t)} k(t, s) u\left(s, x\left(\psi_{3}(s)\right)\right) d s\right), \quad t \geq 0,
\end{aligned}
$$

has been considered very recently by Liang et al. [7].

The main tool used in our research is a measure of weak noncompactness given by Banaś and Knab [3] to find a special subset of $L^{1}[0, \infty)$ and also by applying the Krasnoselskii's fixed point theorem on this set. The existence results generalizing several previous works $[1,8]$ will be proved.

Let us mention that the theory of functional integral equations has many useful applications in describing numerous events and problems of the real world. For example, integral equations are often applicable in engineering, mathematical physics, economics, and biology (cf. [3, 4, 9-12]).

The paper is organized in five sections, including the introduction. Some preliminaries, notations, and auxiliary facts are presented in Section 2; in Section 3, we will introduce the main tools: measure of weak noncompactness and Krasnoselskii's fixed point theorem. The main theorem in our paper will be established in Section 4. In Section 5, we give an example to illustrate our results.

\section{Preliminaries}

Throughout this paper, we let $\mathbb{R}$ be the set of all real numbers, $\mathbb{R}_{+}=[0, \infty)$, and $L^{1}(I)$ denotes the space of the Lebesgue integrable functions on a measurable subset $I$ of $\mathbb{R}$ with the standard norm

$$
\|x\|_{L^{1}(I)}=\int_{I}|x(t)| d t
$$

The space $L^{1}\left(\mathbb{R}_{+}\right)$and $\|x\|_{L^{1}\left(\mathbb{R}_{+}\right)}$will be briefly denoted by $L^{1}$ and $\|\cdot\|$, respectively. Let $I$ be an interval of $\mathbb{R}$ bounded or not.

Definition 1. Consider a function $(t, x, y):=f: I \times \mathbb{R} \times \mathbb{R} \rightarrow$ $\mathbb{R}$. We say that $f$ satisfies Carathéodory conditions if it is measurable in $t$ for any $(x, y) \in \mathbb{R}^{2}$ and continuous in $(x, y)$ for almost all $t \in I$.

Now, we make a short note about the so-called superposition operator, which is one of the simplest and more important operators that are investigated in nonlinear functional analysis, see [13]. Consider a function $f(t, x):=f$ : $I \times \mathbb{R}$. Then for every function $x(t)$ being measurable on $I$, we may assign the function $N_{f}(x)(t)=f(t, x(t)), t \in I$. The operator $N_{f}$ defined in such a way is called the superposition operator generated by the function $f$, for more details about this theory the reader can see $[5,7,14]$. Krasnoselskii [15] and Appell and Zabrejko [12] have proved the following assertion when $I$ is a bounded and an unbounded domain, respectively.
Theorem 2 (see [16]). The superposition operator $N_{f}$ generated by the function $f(t, x)$ maps the space $L^{1}(I)$ continuously into itself if and only if $|f(t, x)| \leq g(t)+c|x|$ for all $t \in I$ and $x \in \mathbb{R}$, where $g$ is a function from the spaces $L^{1}(I)$ and $c \in \mathbb{R}_{+}$.

Theorem 3 (see [17]). Let I be a bounded interval and let $f$ : $I \times \mathbb{R} \rightarrow \mathbb{R}$ be a function satisfying Carathéodory condition. Then it possesses the Scorza-Dragoni property; that is, for each $\varepsilon>0$, there exists a closed subset $D_{\varepsilon}$ of I such that

$$
\text { meas }\left(I \backslash D_{\varepsilon}\right) \leq \varepsilon
$$

and $\left.f\right|_{D_{\varepsilon} \times \mathbb{R}}$ is continuous.

Now, we are going to review a theorem from [14] about the continuity of the linear Volterra integral operator on the space $L^{1}=L^{1}\left(\mathbb{R}_{+}\right)$. Let $\Delta=\{(t, s): 0 \leq s \leq t\}$ and $k: \Delta \rightarrow$ $\mathbb{R}$ be measurable functions with respect to both variables. Consider

$$
(K x)(t)=\int_{0}^{t} k(t, s) x(s) d s, \quad t \in \mathbb{R}_{+}, x \in L^{1}\left(\mathbb{R}_{+}\right) .
$$

We notice that $K$ is a linear Volterra integral operator generated by $k$.

Theorem 4 (see [14]). Let $k$ be measurable on $\Delta$ such that

$$
\text { ess } \sup _{s \geq 0} \int_{s}^{\infty}|k(t, s)| d t<\infty \text {. }
$$

Then the Volterra integral operator $K$ generated by $k$ maps continuously the space $L^{1}\left(\mathbb{R}_{+}\right)$into itself and the norm $\|K\|$ of this operator is majorized by the number ess $\sup _{s \geq 0} \int_{s}^{\infty}|k(t, s)| d t$.

\section{Measure of Weak Noncompactness}

In this section, we collect a few auxiliary facts concerning mainly measures of noncompactness, see [18]. Let $(X,\|\cdot\|)$ be a real Banach space with the zero element $\theta$. Denote by $B(x, r)$ the closed ball centered at $x$ with radius $r$. We will write $B_{r}$ to denote the ball $B(\theta, r)$.

The family of all nonempty and bounded subsets of $X$ will be denoted by $B(X)$, while the subfamily consisting of all relatively weak compact sets is denoted by $W(X)$.

Definition 5 (see [19]). A function $\mu: B(X) \rightarrow \mathbb{R}_{+}$is said to be a measure of weak noncompactness, if it satisfies the following conditions:

(1) the family $\operatorname{ker}(\mu)=\{M \in B(X): \mu(M)=0\}$ is nonempty and $\operatorname{ker}(\mu) \subset W(X)$;

(2) $M_{1} \subset M_{2} \Rightarrow \mu\left(M_{1}\right) \leq \mu\left(M_{2}\right)$;

(3) $\mu\left(C_{0}(M)\right)=\mu(M)$, where $C_{0}(M)$ denotes the convex hull of $M$;

(4) $\mu\left(\lambda M_{1}+(1-\lambda) M_{2}\right) \leq \lambda \mu\left(M_{1}\right)+(1-\lambda) \mu\left(M_{2}\right)$;

(5) if $\left\{M_{n}\right\}_{n}$ is a sequence of nonempty, weak closed subsets of $X$ with $M_{1}$ bounded and $\cdots M_{n} \subset M_{n-1} \subset$ $M_{n-2} \subset \cdots \subset M_{1}$ with $\lim _{n \rightarrow \infty} \mu\left(M_{n}\right)=0$, then $M_{\infty}=\cap_{n=1}^{\infty} M_{n}$ is nonempty. 
The family ker $\mu$ described in (1) is said to be the kernel of the measure of weak noncompactness $\mu$. Observe that the intersection set $M_{\infty}$ from (5) is a member of ker $\mu$. Indeed, since $\mu\left(M_{\infty}\right) \leq \mu\left(M_{n}\right)$ for any $n$ natural, it follows that we get $\mu\left(M_{\infty}\right)=0$. This simple observation will play an important role further on.

We mention that the first important example of measure of weak noncompactness was given by De Blasi [20] with the help of the following formula:

$$
\begin{aligned}
\beta(X)=\inf \{r & >0: \text { there exists a weak compact } \\
& \text { subset } \left.W \text { of } E \text { such that } X \subset W+B_{r}\right\} .
\end{aligned}
$$

The measure of the weak noncompactness $\beta$ has a lot of interesting properties and it is also applied in nonlinear analysis [20].

We observe that it is rather difficult to express the De Blasi measure of weak noncompactness $\beta$ with the help of a convenient and useful formula in a concrete Banach space. Such a formula is only known in the space $L^{1}(I)$, where $I$ is a bounded interval in $\mathbb{R}$ (cf. [21]).

The following nice example of measure of weak noncompactness is a typical measure of weak noncompactness, which was given by Banaś and Knap in [14]. For every nonempty and bounded subset $M$ of the space $L^{1}$, set

$$
\mu(M)=c(M)+d(M),
$$

where

$$
\begin{array}{r}
c(M)=\lim _{\varepsilon \rightarrow 0}\left\{\operatorname { s u p } _ { x \in M } \left\{\operatorname { s u p } \left\{\int_{D}|x(t)| d t: D \subset \mathbb{R}_{+},\right.\right.\right. \\
\operatorname{meas}(D) \leq \varepsilon\}\}\}, \\
d(M)=\lim _{\tau \rightarrow \infty}\left\{\sup \left\{\int_{\tau}^{\infty}|x(t)| d t: x \in M\right\}\right\} ;
\end{array}
$$

then $\mu: B(X) \rightarrow \mathbb{R}_{+}$is a measure of weak noncompactness.

The following results will be used in the sequel.

Theorem 6. A bounded set $Y$ is relatively weakly compact in $L^{1}$ if and only if the following two conditions are satisfied:

(a) for any $\varepsilon>0$ there exists $\delta>0$ such that if meas $(D) \leq$ $\delta$ then $\int_{D}|x(t)| d t \leq \varepsilon$ for all $x \in Y$;

(b) for any $\varepsilon>0$ there exists $\tau>0$ such that $\int_{\tau}^{\infty}|x(t)| d t \leq$ $\varepsilon$ for all $x \in Y$.

Theorem 7 (see [Krasnoselskii's fixed point theorem [22]]). Let $E$ be a closed convex and nonempty subset of a Banach space $X$. Let $F$ and $G$ be two operators such that

(1) $G(E)+F(E) \subset E$,

(2) $G$ is a contraction mapping,

(3) $F(E)$ is relatively compact and $F$ is continuous.

Then there exists $x \in E$ such that $G x+F x=x$.
Lemma 8 (see [23]). Let I be a Lebesgue measurable subset of $\mathbb{R}$ and $1 \leq p \leq \infty$. If $\left\{f_{n}\right\}$ is a convergent sequence to $f \in$ $L^{p}(I)$ in the $L^{p}$-norm, then there is a subsequence $\left\{f_{n_{k}}\right\}$ which converges to $f$ a.e., and there is $g \in L^{p}(I), g \geq 0$, such that

$$
\left|f_{n_{k}}(x)\right| \leq g(x), \quad \text { a.e. } x \in I .
$$

\section{Main Result}

In this section, we consider (1) and we will study the existence of solution under the following assumptions.

(i) The functions $g, f: \mathbb{R}_{+} \times \mathbb{R}^{2} \rightarrow \mathbb{R}$ satisfies Carathéodory conditions and there exist $A, B \in \mathbb{R}_{+}$ and functions $a_{1}, a_{2} \in L^{1}$ such that

$$
\begin{gathered}
|g(t, x, y)| \leq a_{1}(t)+A(|x|+|y|), \quad t \geq 0, x, y \in \mathbb{R}, \\
|f(t, x, y)| \leq a_{2}(t)+B(|x|+|y|), \quad t \geq 0, x, y \in \mathbb{R} .
\end{gathered}
$$

Additionally, the function $g$ satisfies the following Lipschitz condition for almost all $t$ :

$$
\begin{array}{r}
\left|g\left(t, x_{1}, y_{1}\right)-g\left(t, x_{2}, y_{2}\right)\right| \leq C_{1}\left|x_{1}-x_{2}\right|+C_{2}\left|y_{1}-y_{2}\right|, \\
\forall\left(x_{i}, y_{i}\right) \in \mathbb{R} \times \mathbb{R}, i=1,2 .
\end{array}
$$

(ii) The function $u: \mathbb{R}_{+} \times \mathbb{R}_{+} \times \mathbb{R} \rightarrow \mathbb{R}, t \rightarrow u(t, s, x)$, is measurable on $\mathbb{R}_{+}$, for all $(s, x) \in \mathbb{R}_{+} \times \mathbb{R}$. Moreover, the function $(s, x) \rightarrow u(t, s, x)$ is continuous on the set $\mathbb{R}_{+} \times \mathbb{R}$ for each $t \in \mathbb{R}_{+}$.

(iii) $|u(t, s, x)| \leq k_{1}(t, s)+k_{2}(t, s)|x|$ for $(t, s, x) \in \mathbb{R}_{+} \times$ $\mathbb{R}_{+} \times \mathbb{R}$, where $k_{i}: \mathbb{R}_{+} \times \mathbb{R}_{+} \rightarrow \mathbb{R}_{+}(i=$ $1,2)$, satisfies Carathéodory conditions. Moreover, the integral operator $K_{2}$ generated by $k_{2}$, that is,

$$
\left(K_{2} x\right)(t)=\int_{0}^{\psi_{2}(t)} k_{2}(t, s) x\left(\psi_{3}(s)\right) d s,
$$

is a continuous map from $L^{1}$ into itself and $\int_{0}^{\psi_{2}(t)} k_{1}(t, s) d s \in L^{1}$.

(iv) The function $v: \mathbb{R}_{+} \times \mathbb{R}_{+} \times \mathbb{R} \rightarrow \mathbb{R}, t \rightarrow v(t, s, x)$, is measurable on $\mathbb{R}_{+}$, for all $(s, x) \in \mathbb{R}_{+} \times \mathbb{R}$. Moreover, the function $(s, x) \rightarrow v(t, s, x)$ is continuous on $\mathbb{R}_{+} \times$ $\mathbb{R}$ for each $t \in \mathbb{R}_{+}$and $|v(t, s, x)| \leq m(t) n(s)+b(t)|x|$ for $(t, s, x) \in \mathbb{R}_{+} \times \mathbb{R}_{+} \times \mathbb{R}$, where $n, m, b \in L^{1}$.

(v) $\psi_{i}: \mathbb{R}_{+} \rightarrow \mathbb{R}_{+}$are absolutely continuous functions and satisfy $\psi_{i}(D) \subset D$, where $D$ is an arbitrary subset of $\mathbb{R}_{+}$and $1 /\left|\psi_{i}^{\prime}(t)\right| \leq M_{i}$ for all $i \in\{1,2,3,4\}, t \geq 0$.

(vi) $q=A+M_{1} A+B\left\|K_{2}\right\| M_{3}+B M_{4}\|b\|<1$.

We need to the following theorem in the sequel. 
Theorem 9. Under the assumptions (ii), (iii), (iv), and (v), the operators

$$
\begin{gathered}
(U x)(t)=\int_{0}^{\psi_{2}(t)} u\left(t, s, x\left(\psi_{3}(s)\right)\right) d s, \\
(V x)(t)=\int_{0}^{\infty} v\left(t, s, x\left(\psi_{4}(s)\right)\right) d s
\end{gathered}
$$

map $L^{1}$ continuously into itself.

Proof. Let $\left\{x_{n}\right\}$ be an arbitrary sequence in $L^{1}$ which converges to $x$ in $L^{1}$-norm. By using Lemma 8 , there is a subsequence $\left\{x_{n_{k}}\right\}$ which converges to $x$ a.e. and there is $h \in$ $L^{1}$ such that

$$
\left|x_{n_{k}}(s)\right| \leq h(s) \quad \text { a.e. in } \mathbb{R}_{+} .
$$

Since $x_{n_{k}} \rightarrow x$ almost everywhere in $\mathbb{R}_{+}$and $\psi_{3}$ is an absolutely continuous function; then from the continuity of $u$ with respect to the third variable, we get

$$
\begin{aligned}
& u\left(t, s, x_{n_{k}}\left(\psi_{3}(s)\right)\right) \\
& \quad \longrightarrow u\left(t, s, x\left(\psi_{3}(s)\right)\right) \text { for almost all } s, t \in \mathbb{R}_{+}
\end{aligned}
$$

and we have

$$
\left|u\left(t, s, x_{n_{k}}\left(\psi_{3}(s)\right)\right)\right| \leq k_{1}(t, s)+k_{2}(t, s) h\left(\psi_{3}(s)\right) .
$$

Hence, by the Lebesgue dominated convergence theorem, we have

$$
\left(U x_{n_{k}}\right)(t) \longrightarrow(U x)(t) \text { for almost all } t \in \mathbb{R}_{+} .
$$

Inequality (21) implies that

$$
\begin{aligned}
\left|\left(U x_{n_{k}}\right)(t)\right| & \leq \int_{0}^{\psi_{2}(t)}\left|u\left(t, s, x_{n_{k}}\left(\psi_{3}(s)\right)\right)\right| d s \\
& \leq \int_{0}^{\psi_{2}(t)} k_{1}(t, s) d s+\int_{0}^{\psi_{2}(t)} k_{2}(t, s) h\left(\psi_{3}(s)\right) d s,
\end{aligned}
$$

for almost all $t \in \mathbb{R}_{+}$. Regarding the assumptions on $k_{1}$ and $k_{2}$, we obtain

$$
\begin{aligned}
& \int_{0}^{\infty} \int_{0}^{\psi_{2}(t)} k_{1}(t, s) d s d t \\
& \quad+\int_{0}^{\infty} \int_{0}^{\psi_{2}(t)} k_{2}(t, s) h\left(\psi_{3}(s)\right) d s d t<\infty
\end{aligned}
$$

Then from (22), inequalities (23) and (24) and the Lebesgue dominated convergence theorem imply

$$
\left\|U x_{n_{k}}-U x\right\|_{L^{1}} \longrightarrow 0
$$

Since any sequence $\left\{x_{n}\right\}$ converging to $x$ in $L^{1}$ has a convergent subsequence $\left\{x_{n_{k}}\right\}$ such that $U x_{n_{k}} \rightarrow U x$ in $L^{1}$, we can conclude that $U: L^{1} \rightarrow L^{1}$ is a continuous operator.
Similarly, we can prove that $V: L^{1} \rightarrow L^{1}$ is a continuous operator.

Theorem 10. Under assumptions (i)-(vi), the problem (1) has at least one solution $x \in L^{1}$.

Proof. Denote by $F$ the nonlinear Volterra integral operator defined by the formula

$$
\begin{gathered}
(F x)(t)=f\left(t, \int_{0}^{\psi_{2}(t)} u\left(t, s, x\left(\psi_{3}(s)\right)\right) d s,\right. \\
\left.\quad \int_{0}^{\infty} v\left(t, s, x\left(\psi_{4}(s)\right)\right) d s\right), \quad t \geq 0, \\
(G x)(t)=g\left(t, x(t), x\left(\psi_{1}(t)\right)\right) .
\end{gathered}
$$

Then (1) may be written in the following form:

$$
x=G(x)+F(x)
$$

The proof will be given as follows.

We show that $G$ is a contraction. From (i), it follows that

$$
\begin{aligned}
\|G(x)-G(y)\| \leq & C_{1} \int_{0}^{\infty}|x(t)-y(t)| d t \\
& +C_{2} \int_{0}^{\infty}\left|x\left(\psi_{1}(t)\right)-y\left(\psi_{1}(t)\right)\right| d t \\
\leq & C_{1}\|x-y\|+C_{2} M_{1}\|x-y\| \\
\leq & \left(C_{1}+C_{2} M_{1}\right)\|x-y\|=C\|x-y\|, \\
& C=C_{1}+C_{2} M_{1}<1 .
\end{aligned}
$$

From Theorem 9, the operators

$$
\begin{gathered}
(U x)(t)=\int_{0}^{\psi_{2}(t)} u\left(t, s, x\left(\psi_{3}(s)\right)\right) d s, \\
(V x)(t)=\int_{0}^{\infty} v\left(t, s, x\left(\psi_{4}(s)\right)\right) d s
\end{gathered}
$$

map $L^{1}$ continuously into itself; then by using Theorem 2 , the Nemytskii operator generated by $f$ is a continuous operator from $L^{1}$ into itself. Therefore, $F$ is a continuous operator from $L^{1}$ into $L^{1}$.

Now, our aim is to see whether there exists $r>0$ such that $G\left(B_{r}\right)+F\left(B_{r}\right) \subset B_{r}$. For this, let us consider $x$ and $y$ be 
arbitrary functions in $B_{r} \subset L^{1}$ with $r$ to be determined later. In view of our assumptions we get

$$
\begin{aligned}
& \|G(x)+F(y)\| \\
& \leq \int_{0}^{\infty}\left|g\left(t, x(t), x\left(\psi_{1}(t)\right)\right)\right| d t \\
& +\int_{0}^{\infty} \mid f\left(t, \int_{0}^{\psi_{2}(t)} u\left(t, s, y\left(\psi_{3}(s)\right)\right) d s,\right. \\
& \left.\int_{0}^{\infty} v\left(t, s, y\left(\psi_{4}(s)\right)\right) d s\right) \mid d t \\
& \leq \int_{0}^{\infty}\left(a_{1}(t)+A\left(|x(t)|+\left|x\left(\psi_{1}(t)\right)\right|\right)\right) d t \\
& +\int_{0}^{\infty}\left(a_{2}(t)+B\left(\int_{0}^{\psi_{2}(t)}\left|u\left(t, s, y\left(\psi_{3}(s)\right)\right)\right| d s\right.\right. \\
& \left.\left.+\left|v\left(t, s, y\left(\psi_{4}(s)\right)\right) d s\right|\right)\right) d t \\
& \leq\left\|a_{1}\right\|+A\|x\|+M_{1} A\|x\|+\left\|a_{2}\right\| \\
& +B \int_{0}^{\infty}\left(\int_{0}^{\psi_{2}(t)}\left|u\left(t, s, y\left(\psi_{3}(s)\right)\right)\right| d s\right. \\
& \left.+\int_{0}^{\infty}\left|v\left(t, s, y\left(\psi_{4}(s)\right)\right)\right| d s\right) d t \\
& \leq\left\|a_{1}\right\|+A\|x\|+M_{1} A\|x\|+\left\|a_{2}\right\| \\
& +B \int_{0}^{\infty}\left[\int_{0}^{\psi_{2}(t)}\left(k_{1}(t, s)+k_{2}(t, s)\left|y\left(\psi_{3}(s)\right)\right|\right) d s\right. \\
& \left.+\left[\int_{0}^{\infty} m(t) n(s)+b(t)\left|y\left(\psi_{4}(s)\right)\right| d s\right]\right] d t \\
& \leq\left\|a_{1}\right\|+A\|x\|+M_{1} A\|x\|+\left\|a_{2}\right\| \\
& +B \int_{0}^{\infty}\left[\int_{0}^{\psi_{2}(t)} k_{1}(t, s) d s\right] d t \\
& +B \int_{0}^{\infty}\left[\int_{0}^{\psi_{2}(t)} k_{2}(t, s)\left|y\left(\psi_{3}(s)\right)\right| d s\right] \\
& +B \int_{0}^{\infty} m(t)\|n\| d t+B \int_{0}^{\infty} b(t) M_{4}\|y\| d t \\
& \leq\left\|a_{1}\right\|+A\|x\|+M_{1} A\|x\|+\left\|a_{2}\right\| \\
& +B \int_{0}^{\infty}\left[\int_{0}^{\psi_{2}(t)} k_{1}(t, s) d s\right] d t \\
& +B \int_{0}^{\infty}\left[\int_{0}^{\psi_{2}(t)} k_{2}(t, s)\left|y\left(\psi_{3}(s)\right)\right| d s\right] \\
& +B\|n\|\|m\|+M_{4} B\|y\|\|b\|
\end{aligned}
$$

$$
\begin{aligned}
\leq & \left\|a_{1}\right\|+A\|x\|+M_{1} A\|x\|+\left\|a_{2}\right\|+B K_{1} \\
& +B\left\|K_{2}\right\| M_{3}\|y\|+B\|n\|\|m\|+B M_{4}\|y\|\|b\| \\
\leq & \left\|a_{1}\right\|+A r+M_{1} A r+\left\|a_{2}\right\|+B K_{1} \\
& +B\left\|K_{2}\right\| M_{3} r+B\|n\|\|m\|+B M_{4} r\|b\| \\
\leq & r
\end{aligned}
$$

where $K_{1}=\int_{0}^{\infty} \int_{0}^{\psi_{2}(t)} k_{1}(t, s) d s d t$ and $\left\|K_{2}\right\|$ is the norm of the bounded linear operator $\left(K_{2} y\right)(t)=\int_{0}^{\psi_{2}(t)} k_{2}(t, s) y(s) d s$. Hence, from assumption (vi), one can deduce that for $r=$ $\left(\left\|a_{1}\right\|+\left\|a_{2}\right\|+B K_{1}+B\|n\|\|m\|\right) /\left(1-\left(A+M_{1} A+B\left\|K_{2}\right\| M_{3}+\right.\right.$ $\left.\left.\mathrm{BM}_{4}\|b\|\right)\right)$, the operator $G+F$ takes $B_{r}=B(0, r)$ into itself; that is,

$$
G\left(B_{r}\right)+F\left(B_{r}\right) \subset B_{r}
$$

Now, we prove that $\mu(G(M)+F(M)) \leq q \mu(M)$ for all bounded subset $\subset B_{r}$. Take arbitrary numbers $\varepsilon>0$ and $D \subset \mathbb{R}_{+}$such that meas $(D)<\varepsilon$, for any $x, y \in M$; we have

$$
\begin{aligned}
& \int_{D}|(G x+F y)(t)| d t \\
& \leq \int_{D}|(G x)(t)| d t+\int_{D}|(F y)(t)| d t \\
& \leq \int_{D}\left(a_{1}(t)+\left(A|x(t)|+\left|x\left(\psi_{1}(t)\right)\right|\right)\right) d t \\
& \quad+\int_{D}|(F y)(t)| d t \\
& \leq \int_{D}\left(a_{1}(t)+\left(A|x(t)|+\left|x\left(\psi_{1}(t)\right)\right|\right)\right) d t+a_{2}(t) \\
& \quad+B\left|\int_{0}^{\psi_{2}(t)} u\left(t, s, y\left(\psi_{3}(s)\right)\right) d s\right| \\
& \quad+B\left|\int_{0}^{\infty} v\left(t, s, y\left(\psi_{4}(s)\right)\right) d s\right| \\
& \leq \int_{D} a_{1}(t) d t+A \int_{D}|x(t)| d t \\
& \quad+M_{1} A \int_{\psi_{1}(D)}|x(t)| d t+\int_{D} a_{2}(t) d t \\
& +B \int_{D}\left[\int _ { 0 } ^ { \psi _ { 2 } ( t ) } \left[k_{1}(t, s)+k_{2}(t, s)\right.\right. \\
& +B \int_{D}\left[\int_{0}^{\infty}\left[n(s) m(t)+b(t)\left|y\left(\psi_{4}(s)\right)\right|\right] d s\right] d t
\end{aligned}
$$




$$
\begin{aligned}
\leq & \int_{D} a_{1}(t) d t+A \int_{D}|x(t)| d t \\
& +M_{1} A \int_{\psi_{1}(D)}|x(t)| d t \\
& +\int_{D} a_{2}(t) d t+B \int_{D}\left[\int_{0}^{\psi_{2}(t)} k_{1}(t, s) d s\right] d t \\
& +B \int_{D}\left[\int_{0}^{\infty} k_{2}(t, s)\left|y\left(\psi_{3}(s)\right)\right| d s\right] d t \\
& +B \int_{D}\left[\int_{0}^{\infty}\left[n(s) m(t)+b(t)\left|y\left(\psi_{4}(s)\right)\right|\right] d s\right] d t \\
& \left.+B\left[\|n\| \int_{D}|m(t)| d t+M_{4} r\right]_{D}|b(t)| d t\right] . \\
& \left.+B \int_{D}(t) d t+A \int_{D}|x(t)| d t+M_{1} A\right]_{\psi_{1}(D)}|x(t)| d t \\
& +\int_{D}\left|a_{1}(t)\right| d t+A \int_{D}|x(t)| d t \\
& +B \int_{D}\left[\|n\| m(t)+b(t) M_{4} r\right] d t \\
& +B\left\|K_{2}\right\| M_{3} \int_{\psi_{3}(D)}|y(t)| d t \\
& +B \int_{D}\left[\int_{0}^{\psi_{2}(t)} k_{1}(t, s) d s\right] d t
\end{aligned}
$$

Since the set having only one element is also weakly compact, then we have

$$
\begin{array}{rl}
\left(C_{1}\right)=\lim _{\varepsilon \rightarrow 0}\left\{\sup \left[\int_{D}\left|a_{i}(t)\right| d t: \operatorname{meas}(D) \leq \varepsilon\right]\right\}=0 & i=1,2 \\
\left(C_{2}\right) & =\lim _{\varepsilon \rightarrow 0}\left\{\sup \left[\int_{D}|\alpha(t)| d t: \operatorname{meas}(D) \leq \varepsilon\right]\right\}=0, \\
\left(C_{3}\right) & =\lim _{\varepsilon \rightarrow 0}\left\{\sup \left[\int_{D}\left[\int_{0}^{\psi_{2}(t)} k_{1}(t, s) d s\right] d t: \operatorname{meas}(D) \leq \varepsilon\right]\right\} \\
& =0
\end{array}
$$

$$
\begin{aligned}
& \left(C_{4}\right)=\lim _{\varepsilon \rightarrow 0}\left\{\sup \left[\int_{D}|m(t)| d t: \text { meas }(D) \leq \varepsilon\right]\right\}=0 \\
& \left(C_{5}\right)=\lim _{\varepsilon \rightarrow 0}\left\{\sup \left[\int_{D}|b(t)| d t: \operatorname{meas}(D) \leq \varepsilon\right]\right\}=0 .
\end{aligned}
$$

Therefore,

$$
c(G(M)+F(M)) \leq\left(A+M_{1} A+B\left\|K_{2}\right\| M_{3}\right) c(M) .
$$

We conclude that

$$
\begin{aligned}
& c(G(M)+F(M)) \\
& \quad \leq\left(A+M_{1} A+B\left\|K_{2}\right\| M_{3}\right) c(M) \leq q c(M) .
\end{aligned}
$$

For $T>0$ and any $x, y \in M$. By similar calculation, we have

$$
\begin{aligned}
& \int_{T}^{\infty}|G(x)+F(y)| d t \\
& \leq \int_{T}^{\infty} a_{1}(t) d t+A \int_{T}^{\infty}|x(t)| d t \\
& \quad+M_{1} A \int_{\psi_{1}(T)}^{\infty}|x(t)| d t+\int_{T}^{\infty} a_{2}(t) d t \\
& \quad+B \int_{T}^{\infty}\left[\int_{0}^{\psi_{2}(t)} k_{1}(t, s) d s\right] d t \\
& \quad+B\left\|K_{2}\right\| \mathrm{M}_{3} \int_{\psi_{3}(T)}^{\infty}|y(t)| d t \\
& \quad+B \int_{T}^{\infty} \alpha(t)\left[\|n\|+b M_{4} r\right] d t .
\end{aligned}
$$

Since the set having only one element is also weakly compact, then we have

$$
\begin{aligned}
& \left(D_{1}\right)=\lim _{T \rightarrow \infty}\left\{\sup \left[\int_{T}^{\infty}\left|a_{i}(t)\right| d t\right]\right\}=0, \quad i=1,2, \\
& \left(D_{2}\right)=\lim _{T \rightarrow \infty}\left\{\sup \left[\int_{T}^{\infty}|\alpha(t)| d t\right]\right\}=0, \\
& \left(D_{3}\right)=\lim _{T \rightarrow \infty}\left\{\sup \left[\int_{T}^{\infty}\left[\int_{0}^{\psi_{2}(t)} k_{1}(t, s) d s\right] d t\right]\right\}=0, \\
& \left(D_{4}\right)=\lim _{T \rightarrow \infty}\left\{\sup \left[\int_{T}^{\infty}|m(t)| d t\right]\right\}=0, \\
& \left(D_{5}\right)=\lim _{T \rightarrow \infty}\left\{\sup \left[\int_{T}^{\infty}|b(t)| d t\right]\right\}=0 .
\end{aligned}
$$

Therefore,

$$
d(G(M)+F(M)) \leq\left(A+M_{1} A+B\left\|K_{2}\right\| M_{3}\right) d(M) .
$$

Hence, we conclude that

$$
d(G(M)+F(M)) \leq q d(M) .
$$


Therefore, from (35) and (39), we obtain

$$
\mu(G(M)+F(M)) \leq q \mu(M)
$$

for all bounded set contained in $B_{r}$. Let

$$
\begin{aligned}
B_{r}^{1}= & C_{\text {onv }}\left(G\left(B_{r}\right)+F\left(B_{r}\right)\right) \\
& \vdots \\
B_{r}^{n+1} & =C_{\text {onv }}\left(G\left(B_{r}^{n}\right)+F\left(B_{r}^{n}\right)\right), \quad n=1,2, \ldots
\end{aligned}
$$

Then $B_{r}^{n+1}$ and $n=1,2, \ldots$ are nonempty, closed, and convex; so they are weakly closed. Moreover,

$$
\begin{gathered}
B_{r}^{n+1} \subset B_{r}^{n}, \quad n=1,2, \ldots, \\
\mu\left(G\left(B_{r}^{n}\right)+F\left(B_{r}^{n}\right)\right) \leq q \mu\left(B_{r}^{n}\right) \leq \cdots \leq q^{n} \mu\left(B_{r}\right),
\end{gathered}
$$

which yields to

$$
\lim _{n \rightarrow \infty} \mu\left(B_{r}^{n}\right)=0
$$

Define

$$
B_{\infty}=\bigcap_{n=1}^{\infty} B_{r}^{n} .
$$

Then $\mu\left(B_{\infty}\right)=0$. So, by the definition of measure of weak noncompactness, $B_{\infty}$ is nonempty, closed convex and relatively weakly compact. Moreover,

$$
G\left(B_{\infty}\right)+F\left(B_{\infty}\right) \subset B_{\infty}
$$

Now, we prove that $F\left(B_{\infty}\right)$ is relatively compact in $L^{1}$. Let $\left\{x_{n}\right\} \subset B_{\infty}$ be an arbitrary sequence. Since $F\left(B_{\infty}\right) \subset B_{\infty}$, then $\mu\left(F\left(B_{\infty}\right)\right)=0$ which implies that $F\left(B_{\infty}\right)$ and $\left\{x_{n}\right\}$ are relatively weakly compact.

Fix an arbitrary $\varepsilon>0$. Applying Theorem 9, for relatively weakly compact set $F\left(B_{\infty}\right)$, there exists $T>0$ such that

$$
\int_{T}^{\infty}\left|F\left(x_{n}\left(\psi_{3}(t)\right)\right)\right| d t \leq \frac{\varepsilon}{6} .
$$

Considering the functions $\left.f\right|_{[0, T] \times \mathbb{R}_{+} \times \mathbb{R}},\left.u\right|_{[0, T] \times \mathbb{R}_{+} \times \mathbb{R}},\left.a_{i}\right|_{[0, T]}$, $\left.\alpha\right|_{[0, T]}$, and $\left.k_{i}\right|_{[0, T] \times \mathbb{R}_{+}}$, by using Lusin theorem and the generalized version of Scorza-Dragoni theorem, see [24], there exists a closed subset $D_{\varepsilon}$ of interval $[0, T]$ satisfying

$$
\text { meas }\left([0, T] \backslash D_{\varepsilon}\right) \leq \varepsilon,
$$

such that $\left.f\right|_{D_{\varepsilon} \times \mathbb{R}_{+} \times \mathbb{R}},\left.u\right|_{D_{\varepsilon} \times \mathbb{R}_{+} \times \mathbb{R}},\left.a_{i}\right|_{D_{\varepsilon}},\left.b\right|_{D_{\varepsilon}}$, and $\left.k\right|_{D_{\varepsilon} \times \mathbb{R}_{+}},\left.m\right|_{D_{\varepsilon}}$ are continuous. Let us consider an arbitrary $x \in B_{\infty}$. Then for $t \in D_{\varepsilon}$, we have

$$
\begin{aligned}
& \int_{0}^{\psi_{2}(t)} u\left(t, s, x\left(\psi_{3}(s)\right)\right) d s \\
& \leq \int_{0}^{\psi_{2}(t)} k_{1}(t, s) d s \\
& \quad+\int_{0}^{\psi_{2}(t)} k_{2}(t, s)\left|x\left(\psi_{3}(s)\right)\right| d s \\
& \leq \overline{k_{1}} T+\overline{k_{2}} \int_{0}^{\psi_{2}(t)}\left|x\left(\psi_{3}(s)\right)\right| d s \\
& \leq \overline{k_{1}} T+\overline{k_{2}} M_{3} r=: U_{\varepsilon}, \\
& \int_{0}^{\infty} \quad\left(t, s, x\left(\psi_{4}(s)\right)\right) d s \\
& \quad \leq \int_{0}^{\infty}\left(n(s) m(t)+b(t)\left|x\left(\psi_{4}(s)\right)\right|\right) d s \\
& \quad \leq\left(\|n\| \bar{m}+\bar{b} M_{4} r\right)=: V_{\varepsilon},
\end{aligned}
$$

where

$$
\begin{aligned}
\bar{m} & =\sup \left\{|m(t)| ; t \in D_{\varepsilon}\right\}, \\
\overline{k_{i}} & =\sup \left\{\left|k_{i}(t, s)\right| ;(t, s) \in D_{\varepsilon} \times[0, T]\right\}, \\
& \text { for } i=1,2 . \bar{b}=\sup \left\{|b(t)| ; t \in D_{\varepsilon}\right\} .
\end{aligned}
$$

Let us take arbitrary $t_{1}, t_{2} \in D_{\varepsilon}$ and assume that $t_{1}<$ $t_{2}$ without loss of generality. By the monotone increasing property of $\psi_{2}$, we have $\psi_{2}\left(t_{1}\right) \leq \psi_{2}\left(t_{2}\right)$. Therefore, from uniform continuity of $\left.u\right|_{D_{\varepsilon} \times[0, T] \times\left[-U_{\varepsilon},+U_{\varepsilon}\right]}$, we infer that

$$
\begin{aligned}
& \mid \int_{0}^{\psi_{2}\left(t_{2}\right)} u\left(t_{2}, s, x_{n}\left(\psi_{3}(s)\right)\right) \\
& \quad-\int_{0}^{\psi_{2}\left(t_{1}\right)} u\left(t_{1}, s, x_{n}\left(\psi_{3}(s)\right)\right) d s \mid \\
& \leq \mid \int_{0}^{\psi_{2}\left(t_{1}\right)} u\left(t_{2}, s, x_{n}\left(\psi_{3}(s)\right)\right) d s \\
& \quad-\int_{0}^{\psi_{2}\left(t_{1}\right)} u\left(t_{1}, s, x_{n}\left(\psi_{3}(s)\right)\right) d s \mid \\
& \leq \int_{0}^{\psi_{2}\left(t_{2}\right)} u\left(t_{2}, s, x_{n}\left(\psi_{3}(s)\right)\right) d s \mid \\
& -u\left(t_{2}, s, x_{n}\left(\psi_{3}(s)\right)\right) d s \\
& -u\left(t_{1}, s, x_{n}\left(\psi_{3}(s)\right)\right) \mid d s
\end{aligned}
$$




$$
\begin{aligned}
& +\int_{\psi_{2}\left(t_{1}\right)}^{\psi_{2}\left(t_{2}\right)}\left|u\left(t_{2}, s, x_{n}\left(\psi_{3}(s)\right)\right)\right| d s \\
& \leq \int_{[0, T] \backslash D_{\varepsilon}}\left|u\left(t_{2}, s, x_{n}\left(\psi_{3}(s)\right)\right)\right| d s \\
& +\int_{[0, T] \backslash D_{\varepsilon}}\left|u\left(t_{1}, s, x_{n}\left(\psi_{3}(s)\right)\right)\right| d s \\
& +\int_{D_{\varepsilon}} \mid u\left(t_{2}, s, x_{n}\left(\psi_{3}(s)\right)\right) d s \\
& -u\left(t_{1}, s, x_{n}\left(\psi_{3}(s)\right)\right) \mid d s \\
& +\int_{\psi_{2}\left(t_{1}\right)}^{\psi_{2}\left(t_{2}\right)} k_{1}\left(t_{2}, s\right) d s \\
& +\int_{\psi_{2}\left(t_{1}\right)}^{\psi_{2}\left(t_{2}\right)} k_{2}\left(t_{2}, s\right)\left|x_{n}\left(\psi_{3}(s)\right)\right| d s \\
& \leq \omega^{u}\left(\left|t_{2}-t_{1}\right|\right)+2 \overline{k_{1}} \operatorname{meas}\left([0, T] \backslash D_{\varepsilon}\right) \\
& +2 \overline{k_{2}} \int_{[0, T] \backslash D_{\varepsilon}}\left|x_{n}\left(\psi_{3}(s)\right)\right| d s \\
& +\overline{k_{1}}\left|\psi_{2}\left(t_{2}\right)-\psi_{2}\left(t_{1}\right)\right| \\
& +\overline{k_{2}} \int_{\psi_{2}\left(t_{1}\right)}^{\psi_{2}\left(t_{1}\right)}\left|x_{n}\left(\psi_{3}(s)\right)\right| d s \\
& \leq \omega^{u}\left(\left|t_{2}-t_{1}\right|\right)+\overline{k_{1}} \text { meas }\left([0, T] \backslash D_{\varepsilon}\right) \\
& +\overline{k_{2}} M_{3} \int_{\psi_{3}\left([0, T] \backslash D_{\varepsilon}\right)}\left|x_{n}(s)\right| d s \\
& +\overline{k_{1}}\left|\psi_{2}\left(t_{2}\right)-\psi_{2}\left(t_{1}\right)\right| \\
& +\overline{k_{2}} \int_{\psi_{2}\left(t_{1}\right)}^{\psi_{2}\left(t_{2}\right)}\left|x_{n}\left(\psi_{3}(s)\right)\right| d s \\
& \leq \omega^{u}\left(\left|t_{2}-t_{1}\right|\right)+\overline{k_{1}} \text { meas }\left([0, T] \backslash D_{\varepsilon}\right) \\
& +\overline{k_{2}} M_{3} \int_{[0, T] \backslash D_{\varepsilon}}\left|x_{n}(s)\right| d s \\
& +\overline{k_{1}}\left|\psi_{2}\left(t_{2}\right)-\psi_{2}\left(t_{1}\right)\right| \\
& +\overline{k_{2}} \int_{\psi_{2}\left(t_{1}\right)}^{\psi_{2}\left(t_{2}\right)}\left|x_{n}\left(\psi_{3}(s)\right)\right| d s \\
& \leq \omega^{u}\left(\left|t_{2}-t_{1}\right|\right)+\overline{k_{1}} \text { meas }\left([0, T] \backslash D_{\varepsilon}\right) \\
& +\overline{k_{2}} M_{3} r \text { meas }\left([0, T] \backslash D_{\varepsilon}\right) \\
& +\overline{k_{1}}\left|\psi_{2}\left(t_{2}\right)-\psi_{2}\left(t_{1}\right)\right| \\
& +\overline{k_{2}} \int_{\psi_{2}\left(t_{1}\right)}^{\psi_{2}\left(t_{2}\right)}\left|x_{n}\left(\psi_{3}(s)\right)\right| d s \text {, }
\end{aligned}
$$

where $\omega^{u}\left(\left|t_{2}-t_{1}\right|\right)$ denotes the modulus of continuity of $u$ on the set $D_{\varepsilon} \times[0, T] \times\left[-U_{\varepsilon},+U_{\varepsilon}\right]$.

Note that the sequence $\left\{x_{n}\right\} \subset B_{\infty}$ is weakly compact; then take Theorem 6 into account; we infer that the terms of the numerical sequence

$$
\left\{\int_{\psi_{2}\left(t_{1}\right)}^{\psi_{2}\left(t_{2}\right)}\left|x_{n}\left(\psi_{3}(s)\right)\right| d s\right\}
$$

are arbitrary small provided that the numbers $t_{2}-t_{1}$ are small enough. Remark that if $t_{2}-t_{1}$ is small enough, then $\psi_{2}\left(t_{2}\right)-$ $\psi_{2}\left(t_{1}\right)$ is small enough. Then the previous inequality tends to zero independent of $n$ as $t_{2}-t_{1}$ tends to zero. From (48),

$$
\left|\left(U x_{n}\right)(t)\right| \leq \overline{k_{1}} T+\overline{k_{2}} M_{3} r=: U_{\varepsilon} .
$$

Therefore, the sequence $\left\{U x_{n}\right\}$ is equibounded and equicontinuous on the set $D_{\varepsilon}$. Obviously from assumption (49) and similarly to the sequence $\left\{U x_{n}\right\}$, we can easily infer that the sequence

$$
\left(V x_{n}\right)(t)=\int_{0}^{\infty} v\left(t, s, x_{n}\left(\psi_{3}(s)\right)\right) d s
$$

is equibounded and equicontinuous on the set $D_{\varepsilon}$. Hence, uniform continuity of $\left.f\right|_{D_{\varepsilon} \times\left[-U_{\varepsilon}, U_{\varepsilon}\right] \times\left[-V_{\varepsilon}, V_{\varepsilon}\right]}$ implies that the sequence $\left\{F x_{n}\right\}$ is equibounded and equicontinuous on $D_{\varepsilon}$. Then, by Arzela-Ascoli theorem, the sequence $\left\{F x_{n}\right\}$ has a convergent subsequence in the norm $C\left(D_{\varepsilon}\right)$. Therefore, $F\left(B_{\infty}\right)$ is a relatively compact subset of $C\left(D_{\varepsilon}\right)$.

Furthermore, observe that the above reasoning does not depend on the choice of $\varepsilon$. Thus we can construct a sequence $\left\{D_{p}\right\}$ of a closed subsets of the interval $[0, T]$ such that meas $\left([0, T] \backslash D_{p}\right) \rightarrow 0$ as $p \rightarrow \infty$ and such that the sequence $\left\{F x_{n}\right\}$ is relatively compact in every space $C\left(D_{p}\right)$. Passing to subsequences if necessary we can assume that $\left\{F x_{n}\right\}$ is a Cauchy sequence in each space $C\left(D_{p}\right)$, for $p=$ $1,2, \ldots$

Now, by using (46), we have

$$
\int_{T}^{\infty}\left|\left(F x_{n}\right)\left(\psi_{3}(t)\right)-\left(F x_{m}\right)\left(\psi_{3}(t)\right)\right| d t \leq \frac{\varepsilon}{3}
$$

From the relatively weakly compactness of $\left\{F x_{n}\right\}$, we can find $\delta>0$ such that for each closed subset $D$ of the interval $[0, T]$ with meas $([0, T] \backslash D) \leq \delta$, we obtain

$$
\int_{[0, T] \backslash D}\left|\left(F x_{n}\right)\left(\psi_{3}(t)\right)\right| d t \leq \frac{\varepsilon}{6} .
$$

Considering the fact that $\left\{F x_{n}\right\}$ is a Cauchy sequence in $C\left(D_{p}\right)$ for each $p \in \mathbb{N}$. One can find $p_{0}$ such that meas $\left([0, T] \backslash D_{p_{0}}\right) \leq \delta$ and, for $m, n \geq p_{0}$,

$$
\left\|\left(F x_{n}\left(\psi_{3}\right)\right)-\left(F x_{m}\left(\psi_{3}\right)\right)\right\|_{C\left(D_{p_{0}}\right)} \leq \frac{\varepsilon}{3\left(\operatorname{meas}\left(D_{p_{0}}\right)+1\right)} .
$$


Consequently, (56) and (57) imply that

$$
\begin{aligned}
& \int_{0}^{T}\left|\left(F x_{n}\right)\left(\psi_{3}(t)\right)-\left(F x_{m}\right)\left(\psi_{3}(t)\right)\right| d t \\
& \quad=\int_{D_{p_{0}}}\left|\left(F x_{n}\right)\left(\psi_{3}(t)\right)-\left(F x_{m}\right)\left(\psi_{3}(t)\right)\right| d t \\
& \quad+\int_{[0, T] \backslash D_{p_{0}}}\left|\left(F x_{n}\right)\left(\psi_{3}(t)\right)-\left(F x_{m}\right)\left(\psi_{3}(t)\right)\right| d t \leq \frac{\varepsilon}{3}
\end{aligned}
$$

for $m, n \geq p_{0}$.

Now, by considering (55) and the last inequality, we obtain

$$
\left\|\left(F x_{n}\right)-\left(F x_{m}\right)\right\|_{L^{1}} \leq \varepsilon \quad \text { for } m, n \geq k_{0}
$$

which shows that the sequence $\left\{F x_{n}\right\}$ is a Cauchy sequence in the Banach space $L^{1}$. Then $\left\{F x_{n}\right\}$ has a convergent subsequence, which implies that $F\left(B_{\infty}\right)$ is a relatively compact subset of $L^{1}$.

Thus, we conclude by using Krasnoselskii's fixed point theorem that the problem (1) has at least one solution $x \in$ $L^{1}$.

\section{Example}

In this section, we give an example, which can be treated by Theorem 10, but not by the related theorem in [25], since it satisfies the assumptions of Theorem 10, but not fulfill the assumptions of results in [25]. To illustrate the new existence result, we consider the following nonlinear integral equation:

$$
\begin{aligned}
x(t)= & \frac{1}{t^{2}+1}+\frac{1}{6}(x(t)+x(2 t))+\frac{1+t^{2}}{\cosh (t)} \\
& +\int_{0}^{t^{3 / 2}} \exp (-(t+s)) \sin \left(\frac{x(s)}{1+2 t^{2}}\right) d s \\
& +\int_{0}^{\infty} \frac{\ln \left(1+s^{2} x^{2}(4 s)\right)}{3\left(1+t^{2}\right)(s+t+1)} d s, \quad t \geq 0 .
\end{aligned}
$$

Put

$$
\begin{gathered}
g(t, x, y)=\frac{1}{t^{2}+1}+\frac{1}{6}(x+y), \\
|g(t, x, y)| \leq \frac{1}{t^{2}+1}+\frac{1}{6}(|x|+|y|), \\
f(t, x, y)=\frac{1+t^{2}}{\cosh (t)}+x+y, \\
u(t, s, x)=\exp (-(t+s)) \sin \left(\frac{x(s)}{1+2 t^{2}}\right),
\end{gathered}
$$

$$
\begin{aligned}
& v(t, s, x)=\frac{\ln \left(1+s^{2} x^{2}(4 s)\right)}{3\left(1+t^{2}\right)(s+t+1)}, \\
& |v(t, s, x)| \leq \frac{1}{3\left(t^{2}+1\right)} \frac{s|x|}{s+1} \leq \frac{1}{3\left(t^{2}+1\right)}|x|, \\
& \psi_{1}(t)=2 t, \quad a_{1}(t)=\frac{1}{t^{2}+1} \\
& a_{2}(t)=\frac{1+t^{2}}{\cosh (t)}, \quad k_{2}(t, s)=\exp (-(t+s)), \\
& k_{1}(t, s)=0, \\
& |u(t, s, x(s))| \leq k_{2}(t, s)\left|\sin \left(\frac{x(s)}{1+2 t^{2}}\right)\right| \\
& \leq k_{2}(t, s)|x(s)| \\
& \int_{s}^{\infty} \exp (-(t+s)) d t \leq \exp (-2 s) \text {, } \\
& \left\|K_{2}\right\| \leq \sup _{s \geq 0} \int_{s}^{\infty} \exp (-(t+s)) d s \\
& \leq 1 \\
& \psi_{3}(t)=3 t, \quad \psi_{4}(t)=4 t, \\
& \psi_{2}(t)=t^{3 / 2}, \quad\left\|K_{2}\right\| \leq 1, \\
& b(t)=\frac{1}{3\left(t^{2}+1\right)}, \quad\|b\|=\frac{\pi}{6}, \\
& M_{1}=\frac{1}{2}, \quad M_{3}=\frac{1}{4}, \quad M_{4}=\frac{1}{3}, \\
& k_{1}=0, \quad A=\frac{1}{6}, \quad B=1, \quad n=m=0,
\end{aligned}
$$

for $s, t \in \mathbb{R}_{+}$and $x \in \mathbb{R}$. Therefore, we have

$$
\begin{aligned}
q & =A+M_{1} A+B\left\|K_{2}\right\| M_{3}+B M_{4}\|b\| \\
& \leq \frac{1}{6}+\frac{1}{12}+\frac{1}{4}+\frac{\pi}{18} \\
& <1 .
\end{aligned}
$$

All conditions of Theorem 10 are satisfied. Hence, the nonlinear equation (60) has at least one solution in $L^{1}$.

\section{Conflict of Interests}

The authors declare that there is no conflict of interests regarding the publication of this paper.

\section{References}

[1] H. Zhu, "On a nonlinear integral equation with contractive perturbation," Advances in Difference Equations, vol. 2011, Article ID 154742, 10 pages, 2011.

[2] J. Banaś and W. G. El-Sayed, "Measures of noncompactness and solvability of an integral equation in the class of functions of 
locally bounded variation," Journal of Mathematical Analysis and Applications, vol. 167, no. 1, pp. 133-151, 1992.

[3] J. Banaś and Z. Knap, "Integrable solutions of a functionalintegral equation," Revista Matemática de la Universidad Complutense de Madrid, vol. 2, no. 1, pp. 31-38, 1989.

[4] A. O. Arancibia and M. P. Jimenez, $L^{p}$ Solutions of Nonlinear Integral Equations Equadiff 9 CD Brno, Masaryk University, 1997.

[5] P. P. Zabrejko, A. I. Koshelev, M. A. Kranose'skii, S. G. Mikhlin, L. S. Rakovshchik, and V. J. Stecenko, Integral Equations, Noordhoff, Leyden, Netherlands, 1975.

[6] L. Olszowy, "On solutions of functional-integral equations of Urysohn type on an unbounded interval," Mathematical and Computer Modelling, vol. 47, no. 11-12, pp. 1125-1133, 2008.

[7] J. Liang, S.-H. Yan, R. P. Agarwal, and T.-W. Huang, "Integral solution of a class of nonlinear integral equations," Applied Mathematics and Computation, vol. 219, no. 10, pp. 4950-4957, 2013.

[8] M. A. Darwish, "On a perturbed functional integral equation of Urysohn type," Applied Mathematics and Computation, vol. 218, no. 17, pp. 8800-8805, 2012.

[9] M. A. Abdou, A. A. Badr, and M. M. El-Kojok, "On the solution of a mixed nonlinear integral equation," Applied Mathematics and Computation, vol. 217, no. 12, pp. 5466-5475, 2011.

[10] R. P. Agarwal, J. Banaś, B. C. Dhage, and S. D. Sarkate, "Attractivity results for a nonlinear functional integral equation," Georgian Mathematical Journal, vol. 18, no. 1, pp. 1-19, 2011.

[11] R. P. Agarwal and D. O'Regan, Infinite Interval Problems for Differential, Difference and Integral Equations, Kluwer Academic, Dordrecht, The Netherlands, 2001.

[12] J. Appell and P. P. Zabrejko, "Nonlinear superposition operators," in Cambridge Tracts In Mathematics, vol. 95, Cambridge University Press, Cambridge, UK, 1990.

[13] C. Corduneanu, "Nonlinear perturbed integral equations," Revue Roumaine de Mathématiques Pures et Appliquées, vol. 13, pp. 1279-1284, 1968.

[14] J. Banaś and Z. Knap, "Measures of weak noncompactness and nonlinear integral equations of convolution type," Journal of Mathematical Analysis and Applications, vol. 146, no. 2, pp. 353362, 1990.

[15] M. A. Krasnoselskiü, "The continuity of the operator $f u(x)=$ $f[x, u(x)]$," Doklady Akademii Nauk SSRR, vol. 77, pp. 185-188, 1951.

[16] R. Lucchetti and F. Patrone, "On Nemytskii's operator and its application to the lower semicontinuity of integral functionals," Indiana University Mathematics Journal, vol. 29, no. 5, pp. 703713, 1980.

[17] B. Ricceri and A. Villani, "Separability and Scorza-Dragoni's property," Le Matematiche, vol. 37, no. 1, pp. 156-161, 1982.

[18] J. Banaś and K. Goebel, Measures of Noncompactness in Banach Spaces, vol. 60 of Lecture Notes in Pure and Applied Mathematics, Marcel Dekker, New York, NY, USA, 1980.

[19] J. Banaś and J. Rivero, "On measures of weak noncompactness," Annali di Matematica Pura ed Applicata, vol. 151, no. 6, pp. 213224, 1988.

[20] F. S. De Blasi, "On a property of the unit sphere in a Banach space," vol. 21, no. 3-4, pp. 259-262, 1977.

[21] J. Appell and E. de Pascale, "Some parameters associated with the Hausdorff measure of noncompactness in spaces of measurable functions," Bollettino della Unione Matematica Italiana B, vol. 3, no. 2, pp. 497-515, 1984.
[22] M. A. Krasnoselskii, Integral Operators in Space of Summable Functions, Noordhoff, Leyden, The Netherlands, 1976.

[23] H. Brezis, Analyse Fonctionelle Théorie et Applications, Masson, 1983.

[24] C. Castaing, "Une nouvelle extension du théorème de DragoniScorza," Comptes Rendus de l'Académie des Sciences A, vol. 271, pp. A396-A398, 1970.

[25] A. Aghajani, Y. Jalilian, and K. Sadarangani, "Existence of solutions for mixed Volterra-Fredholm integral equations," Electronic Journal of Differential Equations, no. 137, pp. 1-12, 2012. 


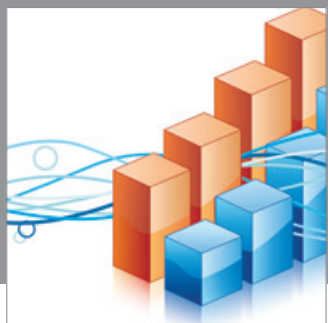

Advances in

Operations Research

mansans

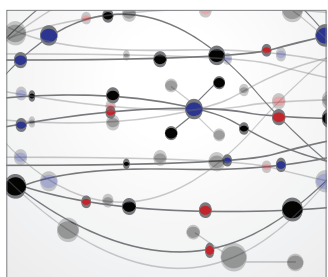

The Scientific World Journal
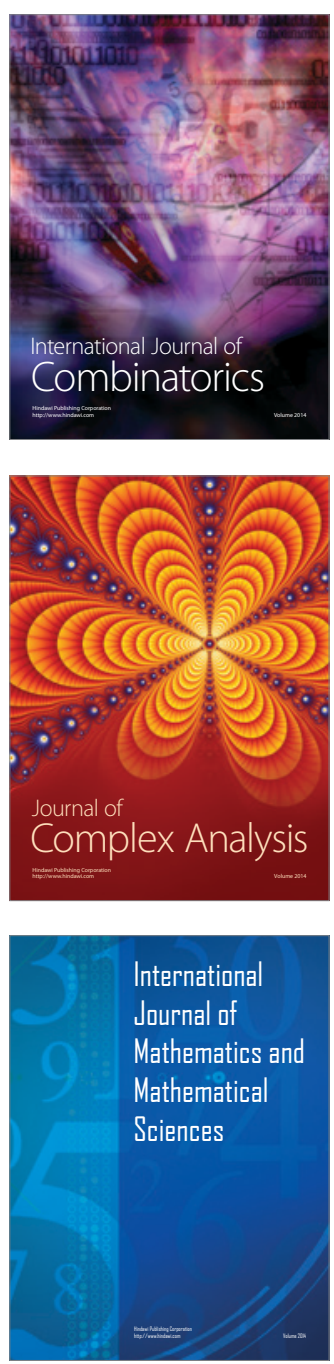
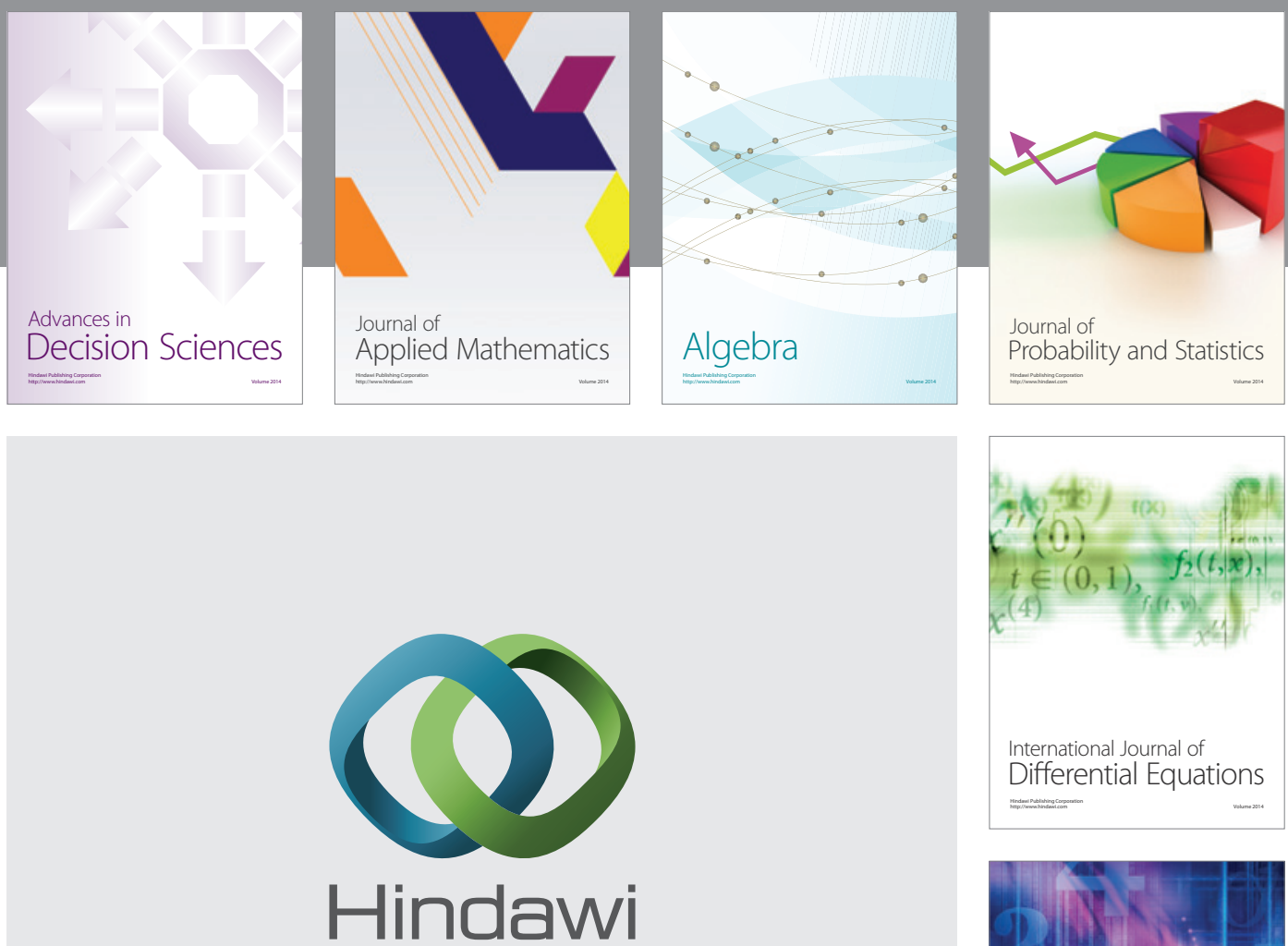

Submit your manuscripts at http://www.hindawi.com
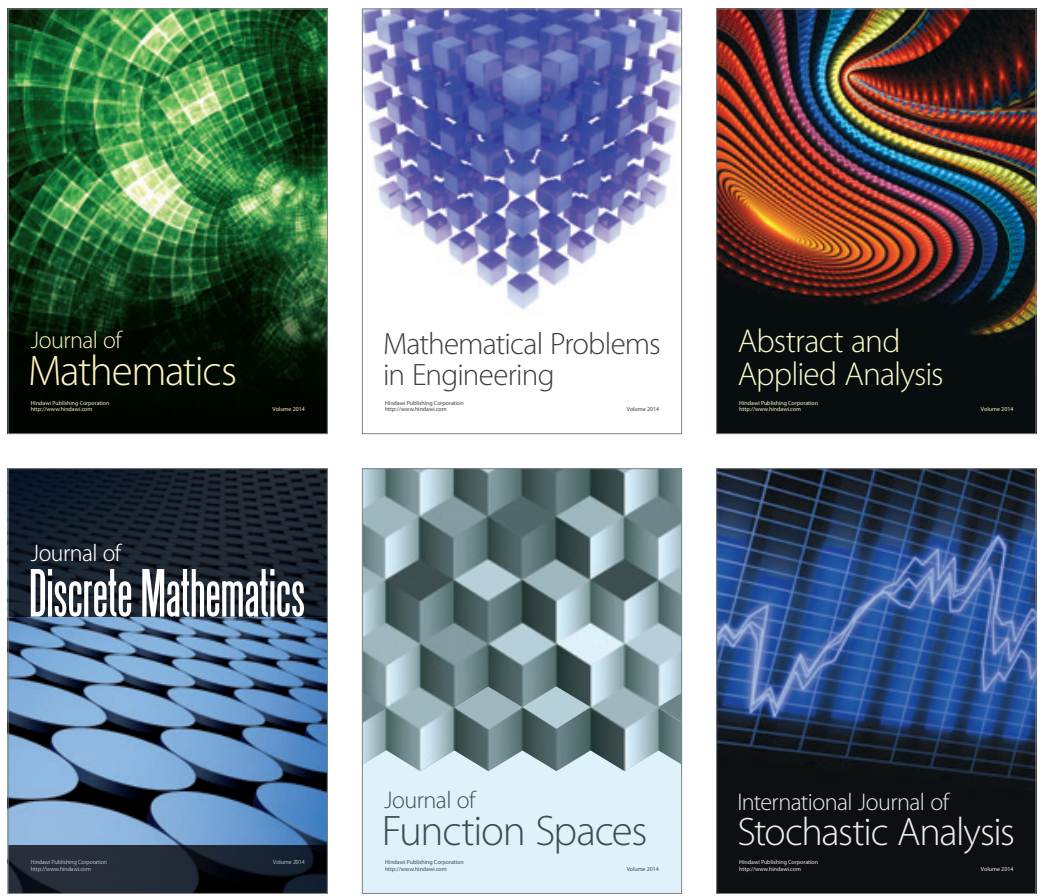

Journal of

Function Spaces

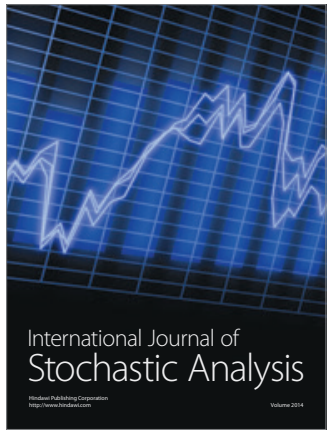

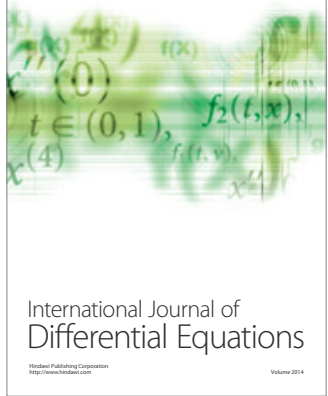
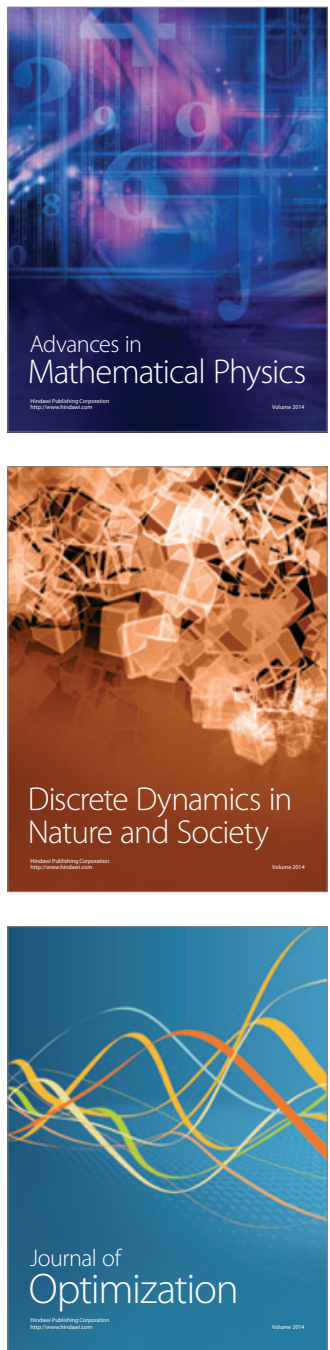